\title{
X Workshop of Physical Agents (WAF'2009)
}

\author{
Pablo Bustos and Pilar Bachiller
}

\begin{abstract}
The Workshop of Physical Agents intends to be a forum for information and experience exchange in different areas regarding the concept of embodied agents, especially applied to the control and coordination of autonomous systems: robots, mobile robots, industrial processes or complex systems. This special issue is devoted to the selected papers presented at the WAF09 that took place from September 10th to 11th in the city of Cáceres (Spain).
\end{abstract}

Index Terms-WAF, Physical Agents.

\section{The 10Th EDITION OF THE Workshop OF PHYSICAL} AGENTS

$\mathbf{T}$ HIS issue of the Journal of Physical Agents (JoPhA) is devoted to the selected papers presented at the $\mathrm{X}$ Workshop of Physical Agents (WAF'2009) that took place from September 10th to 11th in the city of Cáceres (Spain). Since its first celebration in 2000, the Workshop of Physical Agents has favored the meeting of different research groups from different areas of knowledge and with a common interest: physical agents. There are many groups who have taken part in this event and belong and actively participate in RedAF (the Spanish Network of Physical Agents), the main local sponsor organizing this Workshop. This annual event has three main objectives:

- Providing a forum for communication on research, technological innovation and technology transfer in the field of physical agents, encompassing areas such as embedded systems, artificial intelligence, mobile robotics, building automation and ubiquitous computing systems.

- Facilitating communication among researchers from different research groups related to physical agents.

- Providing a means for active and efficient transfer of research and technological upgrading.

In its tenth edition, the Workshop of Physical Agents has successfully achieved these goals once again. We had the participation of different research groups from the national scene who presented the results of their research in areas related to physical agents. We had contributions describing scientific works in different areas that use agent-based technologies concepts, robotics, vision, (software agents) highlighting the similarities and synergies among physical and software agents. This year we also had the participation of Professor Danica Kragic from the Royal Institute of Technology (Stockholm, SWEDEN) who gave the plenary talk Active Vision for Detecting, Fixating, Manipulating Objects and Learning of Human Actions. As in former editions of the workshop, there were shown live demos and exhibitions to assess progress in some research, such as soccer robots and autonomous navigation using vision.

Pablo Bustos and Pilar Bachiller are with University of Extremadura. E-mail: \{pbustos, pilarb\}@unex.es
As a summary of this event, in this publication we include six papers about different topics of vision, SLAM, exploration and applications.

The first one Multi-cue Visual Obstacle Detection for Mobile Robots presents an algorithm for obstacle detection using a stereo camera pair. The method uses both geometric and color cues to perform robustly on heterogeneous circumstances. Authors apply the propose method to the problem of autonomous navigation showing results of real long-term experiments.

The second one Recognition of Standard Platform RoboCup Goals shows a methodology based on artificial vision for goal detection and its application to the RoboCup. The goals are detected by means of color segmentation and geometrical methods. Authors propose methods for estimating the robot position with respect to the goal solving problems as the selflocalization of the robot in the field.

The third paper of this issue Large Scale Egomotion and Error Analysis with Visual Features presents a method for large scale robot $6 \mathrm{DoF}$ movement estimation using stereo images. The method transforms 2D features, based on Sift and Surf detectors, to the 3D space. These features are then used for robot movement correction. The paper shows results of a comparison between the proposed method and the ICP algorithm.

The fourth selected paper Mission Specification in Underwater Robotics describes the utilization of software design patterns and plan-based mission specifications in the definition of missions for autonomous underwater vehicles (AUV). Authors present the main elements of a software framework for programming AUVs, showing a solution to some well-known problems of the development of robotic control software.

The fifth one Balanced Multi-Robot Exploration through a Global Optimization Strategy proposes an original strategy for coordinating multi-robot exploration behaviors. The paper presents an extensive review of the state of the art in multirobot exploration as well as a deep comparative analysis with other exploration techniques.

The last paper of this issue Affine image region detection and description proposes an affine region detector using a hierarchical grouping approach generating an irregular pyramid. In addition, detected regions are characterized by a kernel-based descriptor. Authors present a comparative study showing the performance of the proposal in relation to similar methods.

To conclude this introduction, we would like to thank all the authors for their excellent contributions and to extend our gratitude to all the participants of the X Workshop of Physical Agents. 